Draft VERSION SEPTEMBER 12, 2018

Preprint typeset using $\mathrm{LATEX}_{\mathrm{E}}$ style emulateapj v. 5/2/11

\title{
A HOT JUPITER FOR BREAKFAST? - EARLY STELLAR INGESTION OF PLANETS MAY BE COMMON
}

\author{
Titos Matsakos and Arieh KÖNigl \\ Department of Astronomy \& Astrophysics and The Enrico Fermi Institute, The University of Chicago, Chicago, IL 60637, USA \\ Draft version September 12, 2018
}

\begin{abstract}
Models of planet formation and evolution predict that giant planets form efficiently in protoplanetary disks, that most of these migrate rapidly to the disk's inner edge, and that, if the arriving planet's mass is $\lesssim$ Jupiter's mass, it could remain stranded near that radius. We argue that such planets would be ingested by tidal interaction with the host star on a timescale $\lesssim 1 \mathrm{Gyr}$, and that, in the case of a solar-type host, this would cause the stellar spin to approach the direction of the ingested planet's orbital axis even if the two were initially highly misaligned. Primordially misaligned stars whose effective temperatures are $\gtrsim 6250 \mathrm{~K}$ cannot be realigned in this way because, in contrast with solar-type hosts, their angular momenta are typically higher than the orbital angular momentum of the ingested planet as a result of inefficient magnetic braking and of a comparatively large moment of inertia. Hot Jupiters located farther out from the star can contribute to this process, but their effect is weaker because the tidal interaction strength decreases rapidly with increasing semimajor axis. We demonstrate that, if $\sim 50 \%$ of planetary systems harbored a stranded hot Jupiter, this scenario can in principle account for (1) the good alignment exhibited by planets around cool stars irrespective of the planet's mass or orbital period, (2) the prevalence of misaligned planets around hot stars, (3) the apparent upper bound on the mass of hot Jupiters on retrograde orbits, and (4) the inverse correlation between stellar spin periods and hot-Jupiter masses.
\end{abstract}

Subject headings: planets and satellites: dynamical evolution and stability — planet-star interactions — protoplanetary disks — stars: rotation

\section{INTRODUCTION}

One of the puzzling findings in the study of exoplanets has been the difference in the properties of planets orbiting cool and hot stars. In particular, using measurements of the Rossiter-McLaughlin effect, it has been inferred that the apparent obliquity $\lambda$ (the angle between the stellar spin and planet's orbital angular momentum vectors as projected on the sky) is typically small $\left(\lambda<20^{\circ}\right)$ for cool stars, but that stars of effective temperature $T_{\text {eff }} \gtrsim 6250 \mathrm{~K}$ exhibit a broad range of apparent obliquities, reaching all the way up to $\lambda \sim 180^{\circ}$ (e.g., Winn et al. 2010; Albrecht et al. 2012). It was further found that the masses $M_{\mathrm{p}}$ of planets on apparent retrograde orbits $\left(\lambda>90^{\circ}\right)$ are $<3 M_{\mathrm{J}}$ (where $M_{\mathrm{J}}$ is Jupiter's mass; Hébrard et al. 2011), and that the stellar rotation periods $P_{*}$ of cool stars that host a hot Jupiter (HJ) decrease with increasing $M_{\mathrm{p}}$ (Dawson 2014).

Several models have been advanced to explain the obliquity dichotomy, but so far none can account for all the relevant observations. Interpretations based solely on differences in the stellar properties (e.g., Rogers et al. 2012) cannot address the inferred dependence of the obliquity on $M_{\mathrm{p}}$ and the detection of highly misaligned planets around cool stars. In view of the fact that $T_{\text {eff }} \approx$ $6250 \mathrm{~K}$ corresponds to the temperature above which the size of the outer convective zone of a main-sequence (MS) F star shrinks rapidly, it was suggested that close-in planets in both cool and hot stars are initially distributed over the entire angular range $(0-180$ degrees $)$, but that only in cool stars where a substantial convective envelope is present can a sufficiently massive close-in planet $\left(M_{\mathrm{p}} \geq 1 M_{\mathrm{J}}\right)$ realign the star through tidal interaction (e.g., Winn et al. 2010; Albrecht et al. 2012). The host stars are also subject to magnetic braking, which declines strongly above the same transition temperature (corresponding to the break in the Kraft curve; Kraft 1967), and it was argued (Dawson 2014) that this, rather than the tidal dissipation efficiency, is the main factor underlying the difference in obliquity properties between cool and hot stars. Both variants of this scenario, however, face the conundrum that an HJ undergoing equilibrium tidal interaction with its host star would spiral in and be ingested on a timescale that is comparable to the realignment time. A possible way out of this difficulty is to appeal to the $\{10\}$ component of the dynamical tide, which could in principle significantly reduce the alignment time without affecting the ingestion time (Lai 2012) 1 However, even though this model can be used to account for individual systems (Valsecchi \& Rasio 2014), it remains unclear whether it can explain the overall $\lambda$ distribution of HJs and the manifested difference between cool and hot stars (Rogers \& Lin 2013; Xue et al. 2014). Furthermore, even the basic tidal interaction interpretation of the obliquity dichotomy has now been called into question by the results of Mazeh et al. (2015), who analyzed the rotational photometric modulations of a large sample of Kepler sources and inferred that (1) the conclusion that planets around cool stars are well aligned, and those around hot stars are not, is general and not restricted just to HJs; and (2) the low obliquity of planets around cool stars extends to orbital periods $P_{\text {orb }}$ that are a factor of $\sim 10$ larger than the maximum value $(<4$ days $)$ for robust tidal interaction between an HJ and a $\gtrsim 1$ Gyr-old

1 An alternative possibility, that only the outer convective layer (Winn et al. 2010) - or even just a part of it (Dawson 2014) partakes in the realignment process, is hard to justify on either theoretical or observational grounds. 
G or F star.

In this Letter we propose to address these apparent difficulties and account for many of the observed differences between the properties of planets in cool and hot stars by postulating that, in addition to the tidal interaction with existing close-in planets, a large fraction of the stellar hosts - both cool and hot - ingest a hot Jupiter early on in their evolution. This proposal is motivated by the expectation that a large fraction (up to $80 \%$ according to Trilling et al. 2002) of solar-type stars possess giant planets during their pre-MS phase, and that a large fraction of the giant planets that form in a protoplanetary disk on scales $\lesssim 5$ AU migrate close to their host star before the disk is dispersed (e.g., Ida \& Lin 2004). Numerical simulations incorporating an N-body code and a 1D $\alpha$-viscosity disk model (Thommes et al. 2008) demonstrated that this behavior can be expected for disks with $\alpha \lesssim 0.01$ that are sufficiently massive. The inward planet migration is likely stopped by the strong ( $\gtrsim 1 \mathrm{kG}$ ) protostellar magnetic field that truncates the disk at a radius $\left(r_{\text {in }}\right)$ of a few stellar radii (e.g., Lin et al. 1996). Gravitational interaction with the disk causes a planet reaching $r_{\text {in }}$ to penetrate into the magnetospheric cavity and, if it is massive enough, to undergo eccentricity excitation that can rapidly lead to a collision with the star (e.g., Rice et al. 2008). It was, however, inferred that if $M_{\mathrm{p}}$ is sufficiently small $\left(\lesssim 1 M_{\mathrm{J}}\right)$, the planet would remain stranded at a distance where its orbital period is $\sim 0.5$ of that at $r_{\text {in }}$ until well after the gas disk disappears (on a timescale of $\sim 10^{6}-10^{7} \mathrm{yr}$ ). In our proposed scenario, the primordial disk orientations span a broad angular range that is reflected in the orbital orientations of the stranded planets. When the latter are ingested by tidal interaction with the host star (on a timescale $<1$ Gyr), the absorbed angular momentum is sufficient to align a solar-mass star in that general direction, but not an MS star with $T_{\text {eff }} \gtrsim 6250 \mathrm{~K}$. This is because cool stars have significantly lower angular momenta at the time of ingestion than hot stars as a result of a more efficient magnetic braking process and of a lower moment of inertia. Given the proximity of the stranded HJs (SHJs) to their host stars $\left(P_{\text {orb.SHJ }} \lesssim 2\right.$ days $)$, they can be expected to have been ingested by the time their parent planetary systems are observed; however, giant planets farther out can continue to interact with their host stars and potentially affect their measured obliquities. In our simplified formulation, we model the SHJs using as parameters their characteristic mass $M_{\mathrm{SHJ}}$ and the fraction $p$ of systems that initially harbored an SHJ. By comparing the predictions of this model with the observational data, we infer $M_{\mathrm{SHJ}} \lesssim 1 M_{\mathrm{J}}$ and $p \sim 0.5$.

The potential effect of tidally induced ingestion of HJs on the observed properties of planetary systems has been recognized before. In particular, Jackson et al. (2009) suggested that this process could account for the observed orbital distribution of close-in planets, whereas Teitler \& Königl (2014) proposed that the spinup induced by the deposition of a swallowed planet's orbital angular momentum in the host's envelope can explain the observed dearth of close-in planets around fast-rotating stars. The current proposal further extends this scenario by incorporating not only the distribution of observed planets but also a putative population of HJs that were ingested after being stranded near the inner edges of their associated protoplanetary disks.

\section{MODELING APPROACH}

Using Monte Carlo simulations, we follow the temporal evolution of the stellar and orbital angular momenta of $10^{6}$ systems in the context of an equilibrium-tide model. The Cartesian coordinate frame $(x, y, z)$ is chosen so that the orbital angular momentum $(\boldsymbol{L})$ always points along the $z$ axis and the stellar one $(\boldsymbol{S})$ always lies in the $y$ - $z$ plane. We assume circular orbits and neglect the precession of $\boldsymbol{L}$ and $\boldsymbol{S}$ around the total angular momentum vector 2 The set of equations employed to model the tidal interaction between a star (subscript *) and a planet (subscript p) is:

$$
\begin{gathered}
\frac{d \boldsymbol{L}}{d t}=+\frac{L}{2 \tau_{\mathrm{d}}} \frac{\Omega_{* x}}{\Omega_{\mathrm{orb}}} \hat{\boldsymbol{x}}+\frac{L}{2 \tau_{\mathrm{d}}} \frac{\Omega_{* y}}{\Omega_{\mathrm{orb}}} \hat{\boldsymbol{y}}-\frac{L}{\tau_{\mathrm{d}}}\left(1-\frac{\Omega_{* z}}{\Omega_{\mathrm{orb}}}\right) \hat{\boldsymbol{z}},(1) \\
\frac{d \boldsymbol{S}}{d t}=-\frac{L}{2 \tau_{\mathrm{d}}} \frac{\Omega_{* x}}{\Omega_{\mathrm{orb}}} \hat{\boldsymbol{x}}-\frac{L}{2 \tau_{\mathrm{d}}} \frac{\Omega_{* y}}{\Omega_{\mathrm{orb}}} \hat{\boldsymbol{y}}+\frac{L}{\tau_{\mathrm{d}}}\left(1-\frac{\Omega_{* z}}{\Omega_{\mathrm{orb}}}\right) \hat{\boldsymbol{z}}-\boldsymbol{T},
\end{gathered}
$$

where $\boldsymbol{\Omega}_{\text {orb }}=\Omega_{\text {orb }} \hat{\boldsymbol{z}}$ and $\boldsymbol{\Omega}_{*}$ are the orbital and stellar angular velocities, respectively (related to the orbital and stellar rotation periods through $P_{\text {orb }}=2 \pi / \Omega_{\text {orb }}$ and $\left.P_{*}=2 \pi / \Omega_{*}\right), \boldsymbol{T}=T \hat{\boldsymbol{S}}$ is the magnetic braking torque, and $\tau_{\mathrm{d}}$ is the nominal tidal damping time, which, neglecting dissipation inside the planet, is given by

$$
\tau_{\mathrm{d}}=\frac{4 Q_{*}^{\prime}}{9}\left(\frac{a}{R_{*}}\right)^{5} \frac{M_{*}}{M_{\mathrm{p}}} \frac{1}{\Omega_{\mathrm{orb}}} .
$$

In Equation (3), $Q_{*}^{\prime}$ is the tidal quality factor (taken to be a constant, for simplicity), $a$ is the semimajor axis, whereas $R$ and $M$ denote an object's radius and mass, respectively. We model the star as a uniformly rotating body $\left(S=I_{*} \Omega_{*}\right)$ with a moment of inertia $I_{*} \approx 0.06 M_{*} R_{*}^{2}$, and adopt for $T$ the expression presented by Matt et al. (2015), which covers both the saturated and the unsaturated regimes and incorporates as a key variable the convective turnover timescale as a function of $T_{\text {eff }}\left(3300 \leq T_{\text {eff }} \leq 7000\right.$; Cranmer \& Saar 2011) . The nominal magnetic braking time is $\tau_{\mathrm{mb}}=|S / T|$.

The equations are solved in a two-step process: we first integrate for a small $\Delta t$ employing a 4th-order RungeKutta method and then rotate the vectors so that the updated $\boldsymbol{L}$ is along $\hat{\boldsymbol{z}}$ and the updated $\boldsymbol{S}$ lies in the $y$ - $z$ plane. When considering a multiplanet system, we solve the equations for the different planets in sequence at each time step, neglecting possible interactions between them and assuming that their orbits remain coplanar.

We consider two sets of models based on the type of host star: G or F; see Table 1 for a listing of the adopted parameters. For each stellar type, we evolve $5 \times 10^{5}$ systems, in each case considering simultaneously the SHJs (parametrized by $M_{\mathrm{SHJ}}$ and $p$; see Section [1) as well as 5 additional planets 3 for which we randomly pick the orbital periods, planet radii, and system age according to the procedure described in Teitler \& König]

2 The associated torques act along the $x$ axis and thus do not affect either the orbital separation or the alignment.

3 Our results are not sensitive to the choice of the number of additional planets because large, close-in planets — which, according to Equation (3), exert the strongest tidal effect - are a rare occurrence. 
TABLE 1

Model Parameters

\begin{tabular}{lcc}
\hline \hline \multicolumn{1}{c}{ Parameter } & Value $(\mathrm{G} / \mathrm{F})$ & Distribution \\
\hline$T_{\text {eff }}[\mathrm{K}]$ & $5500 / 6400$ & \\
$M_{*}\left[M_{\odot}\right]$ & $1.0 / 1.3$ & \\
$R_{*}\left[R_{\odot}\right]$ & $1.0 / 1.3$ & \\
$P_{* \text { ini }}[$ days $]$ & $5-10 / 2.5-5^{\mathrm{a}}$ & Uniform \\
Age $[\mathrm{Gyr}]$ & $1-8 / 1-4^{\mathrm{b}}$ & Fit to data \\
\hline Number of planets & 5 & \\
$P_{\text {orb }}[$ days $]$ & $0.5-50^{\mathrm{c}}$ & $f(\ln P) \propto P^{0.54}$ \\
$R_{\mathrm{p}}\left[R_{\oplus}\right]$ for $P_{\text {orb }}<7 \mathrm{~d}$ & $2-20^{\mathrm{c}}$ & $f\left(\ln R_{\mathrm{p}}\right) \propto R_{\mathrm{p}}^{-1.09}$ \\
$R_{\mathrm{p}}\left[R_{\oplus}\right]$ for $P_{\text {orb }}>7 \mathrm{~d}$ & $2-20^{\mathrm{c}}$ & $f\left(\ln R_{\mathrm{p}}\right) \propto R_{\mathrm{p}}^{-2.31}$ \\
$M_{\mathrm{p}}\left[M_{\oplus}\right]$ for $R_{\mathrm{p}}<9 R_{\oplus}$ & $4-81^{\mathrm{d}}$ & $\left(R_{\mathrm{p}} / R_{\oplus}\right)^{2} M_{\oplus}$ \\
$M_{\mathrm{p}}\left[M_{\oplus}\right]$ for $R_{\mathrm{p}}>9 R_{\oplus}$ & $56-5620^{\mathrm{d}}$ & Fit to data \\
$\psi_{\text {ini }}[$ degrees $]($ random $)$ & $0-180$ & $f(\psi) \propto \sin (\psi)$ \\
\hline$p$ (SHJ fraction) & 0.5 & \\
$M_{\mathrm{SHJ}}\left[M_{\mathrm{J}}\right]$ & 0.6 & \\
$R_{\mathrm{SHJ}}\left[R_{\mathrm{J}}\right]$ & 1.0 & \\
$P_{\text {orb }, \mathrm{SHJ}}[$ days $]$ & 2.0 & \\
\hline
\end{tabular}

References. - ${ }^{\mathrm{a}}$ Meibom et al. (2011); ${ }^{\mathrm{b}}$ Walkowicz \& Basri (2013); ${ }^{\mathrm{c}}$ Youdin (2011); d Weiss et al. (2013)).

Note. - The subscript "ini" indicates an initial value.

(2014) 4 As in that work, we adopt the approximation that the entire planetary orbital angular momentum is added to $S$ when the planet reaches the Roche limit $a_{\mathrm{R}} \approx 1.5\left(3 M_{*} / M_{\mathrm{p}}\right)^{1 / 3} R_{\mathrm{p}}$, and we neglect stellar evolution effects (but see, e.g., Valsecchi et al. 2015 and Valsecchi \& Rasio 2014). The effective value of $Q_{*}^{\prime}$ and its physical basis are still open questions (e.g., Ogilvie 2014). Note, however, that the apparent break at $P_{\text {orb }} \gtrsim$ 3 days in the period distribution of planets around solarmass stars (e.g., Youdin 2011), if due to tidal interaction, is consistent with $Q_{*}^{\prime} \approx 10^{6}$ (e.g., Teitler \& König] 2014). Using the data from exoplanets.org, we find that F-star systems manifest a similar break at the same location. We therefore adopt $Q_{*}^{\prime} \sim 10^{6}$ for both our cool and hot fiducial stars.

The initial spin-orbit angle $\psi_{\text {ini }}$ is taken to be random, $f\left(\psi_{\text {ini }}\right) \propto \sin \left(\psi_{\text {ini }}\right)$, which corresponds to a flat distribution for the projected angle $\lambda_{\text {ini }}$ (see Fabrvcky \& Winn 2009). Our choice of $P_{\text {orb,SHJ }} \approx 2$ days is motivated by the characteristic period ( $\sim 7$ days; Gallet \& Bouvier 2013) of disk-accreting protostars, which can be interpreted in terms of the magnetic disk-locking model (e.g., Königl 1991). In this picture, the disk truncation radius is located at $r_{\text {in }} \approx 0.7 r_{\text {co }}$ (with $r_{\text {co }}$ defined by $\left.\Omega_{\mathrm{orb}}\left(r_{\mathrm{co}}\right)=\Omega_{*}\right)$ if the magnetic interaction is dominated by the dipolar field component (Long et al. 2005). The choice of $P_{\text {orb,SHJ }}$ then follows from the results of Rice et al. (2008), who inferred that it is $\sim 0.5 P_{\text {orb }}\left(r_{\text {in }}\right) 5$

\section{RESULTS}

The results for the final configurations of the modeled planetary systems are presented in identical formats in

4 In relating planet masses to planet radii, we generalized the treatment in Teitler \& König] (2014) by employing a power-law relationship only for $R_{\mathrm{p}}<9 R_{\oplus}$ and using an empirical fit to the data for $R_{\mathrm{p}}>9 R_{\oplus}$; see Table 1

5 Using the numerical results of Long et al. (2005) and the characteristic radius $\left(\sim 2 R_{\odot}\right)$ and mass accretion rate $(\sim$ $10^{-8} M_{\odot} \mathrm{yr}^{-1}$ ) for classical $\mathrm{T}$ Tauri stars, we infer a stellar dipolar field strength of $\sim 2.5 \mathrm{kG}$, which is consistent with observations (e.g., Johns-Krull 2007).
TABLE 2

SHJ INGESTION

\begin{tabular}{l|ccc|ccc}
\hline \hline Parameter & \multicolumn{3}{|c|}{ G star } & \multicolumn{3}{c}{ F star } \\
& Init. & Roche & Ingest. & Init. & Roche & Ingest. \\
\hline$t[\mathrm{Gyr}]$ & 0.0 & 0.69 & 0.69 & 0.0 & 0.35 & 0.35 \\
$a[\mathrm{AU}]$ & 0.03 & 0.01 & - & 0.03 & 0.01 & - \\
$P_{\text {orb }}[$ days $]$ & 2 & 0.5 & - & 2 & 0.5 & - \\
$P_{*}[$ days $]$ & 7.5 & 12.9 & 5.0 & 3.8 & 4.5 & 4.2 \\
$\tau_{\text {mb }}[\mathrm{Gyr}]$ & 0.21 & 0.61 & 0.09 & 1.96 & 2.83 & 2.46 \\
$\left|L_{\text {SHJ }} / S\right|$ & 1.59 & 1.71 & - & 0.43 & 0.33 & - \\
$\psi[$ degrees $]$ & 100 & 35 & 13 & 100 & 86 & 69 \\
\hline
\end{tabular}

Figures 1 and 2 for our representative $G$ and $F$ stars, respectively. The top three panels show the probability distribution function of the projected spin-orbit angle for the entire population (left) and separately for its short-period $\left(P_{\text {orb }}<5\right.$ days, middle) and long-period ( $P_{\text {orb }}>5$ days, right) components. These panels demonstrate that our model can account for the basic trends uncovered by the Mazeh et al. (2015) study - that planets around cool stars are well aligned with their host's spin irrespective of the planet's size or orbital period, but that planets around hot stars are not. To quantify the degree of alignment, we follow Mazeh et al. (2015) and evaluate the ratio $q \equiv\left\langle\sin \left(i_{*}\right)\right\rangle /(\pi / 4)$, where $i_{*} \in[0, \pi / 2]$ is the angle between the stellar rotation axis and the line of sight, the angle brackets denote an average over the distribution, and the normalization is by the value of $\left\langle\sin \left(i_{*}\right)\right\rangle$ for a random orientation of $\boldsymbol{S}$ on the plane of the sky. This ratio is therefore 1 when there is no preferred spin-orbit orientation (as in our adopted initial condition), whereas it is $q=1 /(\pi / 4) \simeq 1.273$ when $\boldsymbol{S}$ is normal to the line of sight (which, in the case of a transiting planet, likely corresponds to $\lambda \approx 0$ ). For our G-host model we obtain $q=1.145$, which corresponds to having the $\sim 50 \%$ of systems that ingested an SHJ attain good alignment even as the rest remain close to their initial random orientations. For the F-host systems we find $q=1.030$, indicating that in this case the initial random distribution is only slightly modified. We note from Figure 1 of Mazeh et al. (2015) that, while the median value of $q$ for stars with $T_{\text {eff }} \lesssim 5000 \mathrm{~K}$ is consistent with perfect alignment, this ratio decreases toward 1 in the G-star range $\left(T_{\text {eff }} \sim 5300-6000 \mathrm{~K}\right)$ that we have modeled 6

The bottom three panels of Figures 1 and 2 confront our model predictions for HJs (defined here by $M_{\mathrm{p}}>$ $0.5 M_{\mathrm{J}}$ and $P_{\mathrm{orb}}<10$ days) with the observational data (as of June 2015) obtained from exoplanets .org, which consist of $N_{\mathrm{G}}=36$ entries for cool stars $\left(T_{\mathrm{eff}}<6250 \mathrm{~K}\right)$ and $N_{\mathrm{F}}=19$ entries for hot ones $\left(6250 \mathrm{~K}<T_{\text {eff }}<\right.$ $7000 \mathrm{~K})$. The left panel shows the planet counts as a function of $\lambda$, with the model predictions obtained by selecting 1000 samples from our simulations, each consisting of $N_{\mathrm{G}}$ (Figure 1) or $N_{\mathrm{F}}$ (Figure 2) systems, and plotting the average counts per bin (bars) as well as the range of \pm 1 standard deviation (shaded areas). It is seen that the model is in excellent agreement with the data,

6 Mazeh et al. (2015) inferred $q<1$ for their hot-star sample, a value that cannot be explained in the context of our basic model. Note in this connection that, as discussed by these authors, the observational challenges that need to be overcome in deriving the hot-star result are more severe than those encountered in the coolstar case. 
with the only $>1 \sigma$ deviation exhibited by the bin encompassing $\lambda=180^{\circ} 7$

The key feature that enables our model to account for the aforementioned observations is the ingestion, over time intervals $\left(t_{\text {ingest }}\right)$ that are shorter than the ages of the observed systems, of SHJs with values of $\left|L_{\mathrm{SHJ}} / S\right|$ that are $>1$ for $\mathrm{G}$ stars and $<1$ for $\mathrm{F}$ stars (see Table 2). The low obliquity attained by initially misaligned $\mathrm{G}$ stars at $t_{\text {ingest }}$ is due mainly to the efficient reduction of $S$ through magnetic braking over the time interval $t_{\text {ingest }}$; by contrast, $\tau_{\mathrm{mb}}\left(t \leq t_{\text {ingest }}\right)>t_{\text {ingest }}$ for $\mathrm{F}$ stars. The strongest constraints on the parameters of the postulated SHJ population are provided by the observed $\lambda$ distributions of HJs for the G- and F-star systems. In particular, by comparing the number counts of well-aligned HJs in solar-type stars (corresponding to the first bin in the lower left panel of Figure1) with those in the $\lambda>20^{\circ}$ bins, one infers $p \sim 0.5$ : the value of this parameter can be expected to reflect both the formation rate and radial transport properties of HJs in protoplanetary disks and the fraction of systems in which disk truncation is either short-lived or inefficient (e.g., Herbst \& Mundt 2005). The corresponding data for F stars (lower left panel of Figure 2) in turn imply an upper bound $\left(\sim 1 M_{\mathrm{J}}\right)$ on $M_{\mathrm{SHJ}}$ : If the SHJ mass were measurably larger, there would be significantly more aligned HJs detected in these systems. Interestingly, this upper bound is consistent with the limit obtained in the SHJ formation scenario studied by Rice et al. (2008). We emphasize that the qualitative results of our model are not sensitive to the exact values of these (or any of the other) parameters. Additional data would, however, be useful for better constraining these values.

The host stars also interact tidally with HJs that are still orbiting (and thus remain observable), which typically have $P_{\text {orb }}$ values of a few days. While the impact of this interaction is not as pronounced as that of ingestion, it can still affect the observed properties of these systems. This is illustrated in the middle and right bottom panels of Figures 1 and 2 which display, respectively, the dependence of $\lambda$ and of the projected stellar angular velocity on the HJ mass for a random selection of 100 simulated systems. It was already recognized before that the tidal interaction scenario is consistent with the apparent dearth of massive planets with either retrograde orbits or high- $P_{*}$ hosts 8 This follows from the inverse dependence of $\tau_{\mathrm{d}}$ (Equation (3)) on $M_{\mathrm{p}}$, which implies that more massive HJs should be more efficient at realigning and spinning up their hosts. The displayed results demonstrate that our model also broadly reproduces these trends quantitatively, for both $\mathrm{G}$ and $\mathrm{F}$ stars.

\section{DISCUSSION}

Our interpretation of the good alignment inferred for long-period planets around cool stars (Mazeh et al. 2015) relies on the orbital planes of the ingested SHJ and of any remaining distant planet having roughly the same orientation, which is consistent with the picture of giant planets forming in a nearly planar disk and migrating to the vicinity of the host star. However, this scenario is not consistent with interpretations of primordial misalignment in terms of either planet-planet scattering (e.g., Beaugé \& Nesvorný 2012) or a KozaiLidov-type interaction with a misaligned companion (e.g., Fabrycky \& Tremaine 2007), as these mechanisms typically result in different orientations for the orbit of an HJ and those of more distant planets. There have already been several proposals in the literature for forming protoplanetary disks that are misaligned with the protostellar spin axis, including a warping torque exerted by the stellar magnetic field (Lai et al. 2011); a gravitational torque exerted by a misaligned companion (Batygin 2012), possibly amplified by a resonance between the disk-torquing frequency and the (diskdriven) stellar precession frequency (Batygin \& Adams 2013); a combination of the above two torques (Lail 2014; Spalding \& Batygin 2014); and accretion from a turbulent interstellar medium (Bate et al. 2010; Fielding et al. 2015). However, these models have not yet been fully evaluated in light of the observed $\lambda$ distribution of transiting planets (e.g., Crida \& Batygin 2014) and other observational constraints (e.g., Watson et al. 2011; Greaves et al. 2014). In this work we adopted a completely random distribution of obliquities for simplicity, but more data and improved modeling might be able to constrain $f\left(\psi_{\text {ini }}\right)$ in the context of this scenario. Future steps toward refining our model could include taking account of stellar evolution and of the effect of dynamical tides, incorporating an inner boundary condition that represents the stellar magnetosphere into global models of planet formation and evolution to better predict the properties of SHJs, and reconciling the SHJ formation picture with the observed distribution of lower-mass planets (e.g., Mandell et al. 2007; Fogg \& Nelson 2007).

The clearest observational prediction of this model is the occurrence of highly misaligned HJs with $P_{\text {orb }} \lesssim$ 2 days around very young stars, but finding such objects could be rather challenging (e.g., Miller et al. 2008; Crockett et al. 2012). There is at present only one claimed detection of a short-period ( $P_{\text {orb }} \approx 0.45$ days $)$, Jupiter-mass planet around a pre-MS $(\sim 3 \mathrm{Myr})$, lowmass star (van Eyken et al. 2012), and it has, in fact, been inferred to be highly misaligned $\left(\lambda \sim 70^{\circ}\right)$; however, the true nature of this object is still being debated (e.g., Ciardi et al. 2015 and references therein).

We are grateful to Dan Fabrycky, Tsevi Mazeh, Fred Rasio, Seth Teitler, Francesca Valsecchi, and Joshua Winn for fruitful discussions. This work was supported in part by NASA ATP grant NNX13AH56G.

\section{REFERENCES}

7 One possible explanation of the observed excess - if indeed it is significant - is that it arises from the action of the $\{10\}$ component of the dynamical tide induced by surviving HJs, which has been neglected in our treatment (see Xue et al. 2014).

8 The decrease of $P_{*}$ with increasing $M_{\mathrm{p}}$ was noted in the case of cool stars by Dawson (2014); however, as the data shown in
Albrecht, S., Winn, J. N., Johnson, J. A., et al. 2012, ApJ, 757, 18 Bate, M. R., Lodato, G., \& Pringle, J. E. 2010, MNRAS, 401, 1505

Batygin, K. 2012, Nature, 491, 418

Figure 2 suggest, it also characterizes hot stars. 

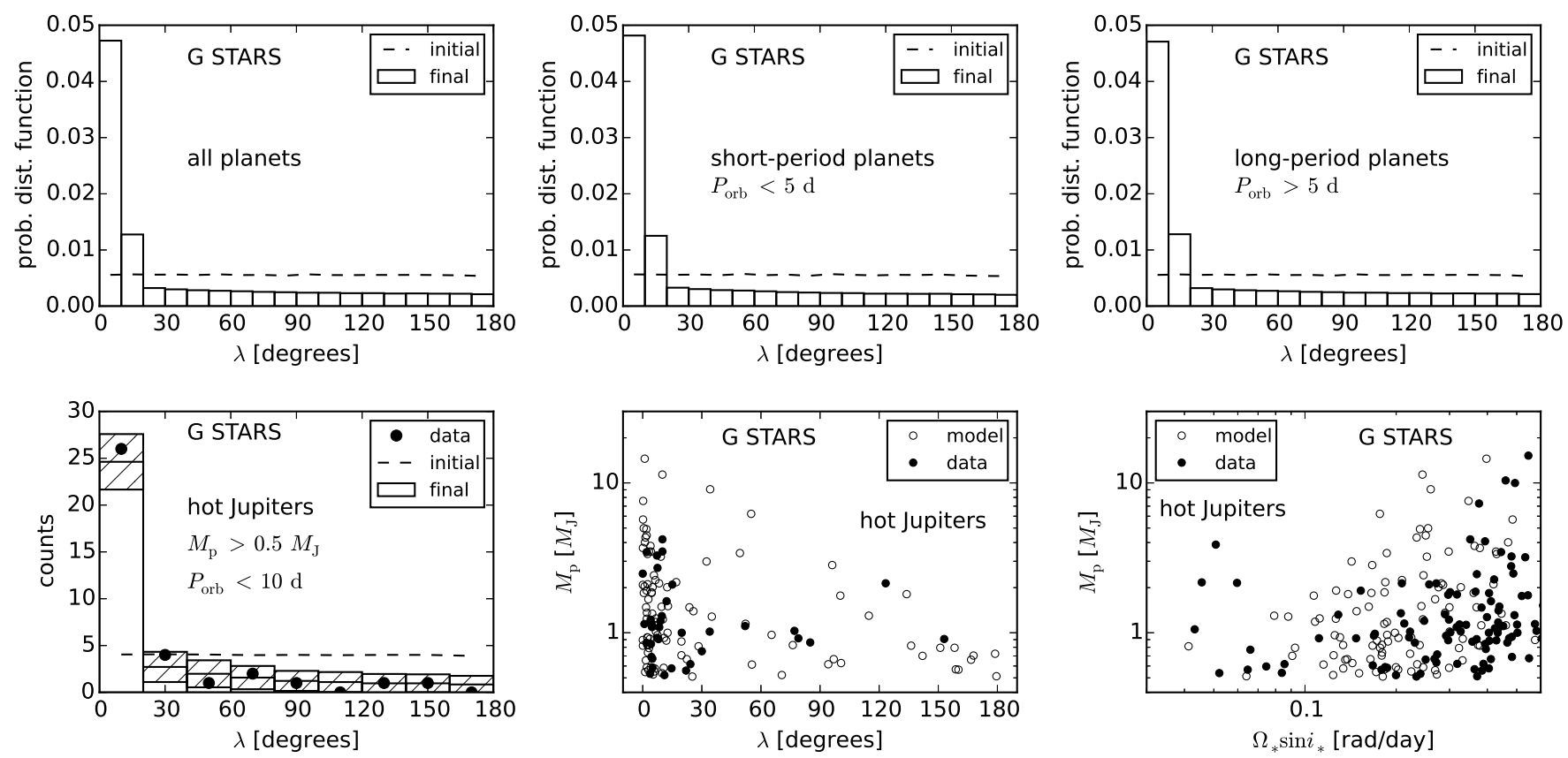

FIG. 1. - System properties in the cool-star model, as functions of either the projected obliquity $\lambda$ or the projected stellar angular velocity. The top panels include the entire range of planet masses and show the $\lambda$ probability distribution for all the planets (top left) and separately for those with $P_{\text {orb }}<5$ days (top middle) and $P_{\text {orb }}>5$ days (top right). The bottom panels show results for hot Jupiters only, along with observational data taken from exoplanets.org: the lower left displays planet number counts and the other two the planet mass.
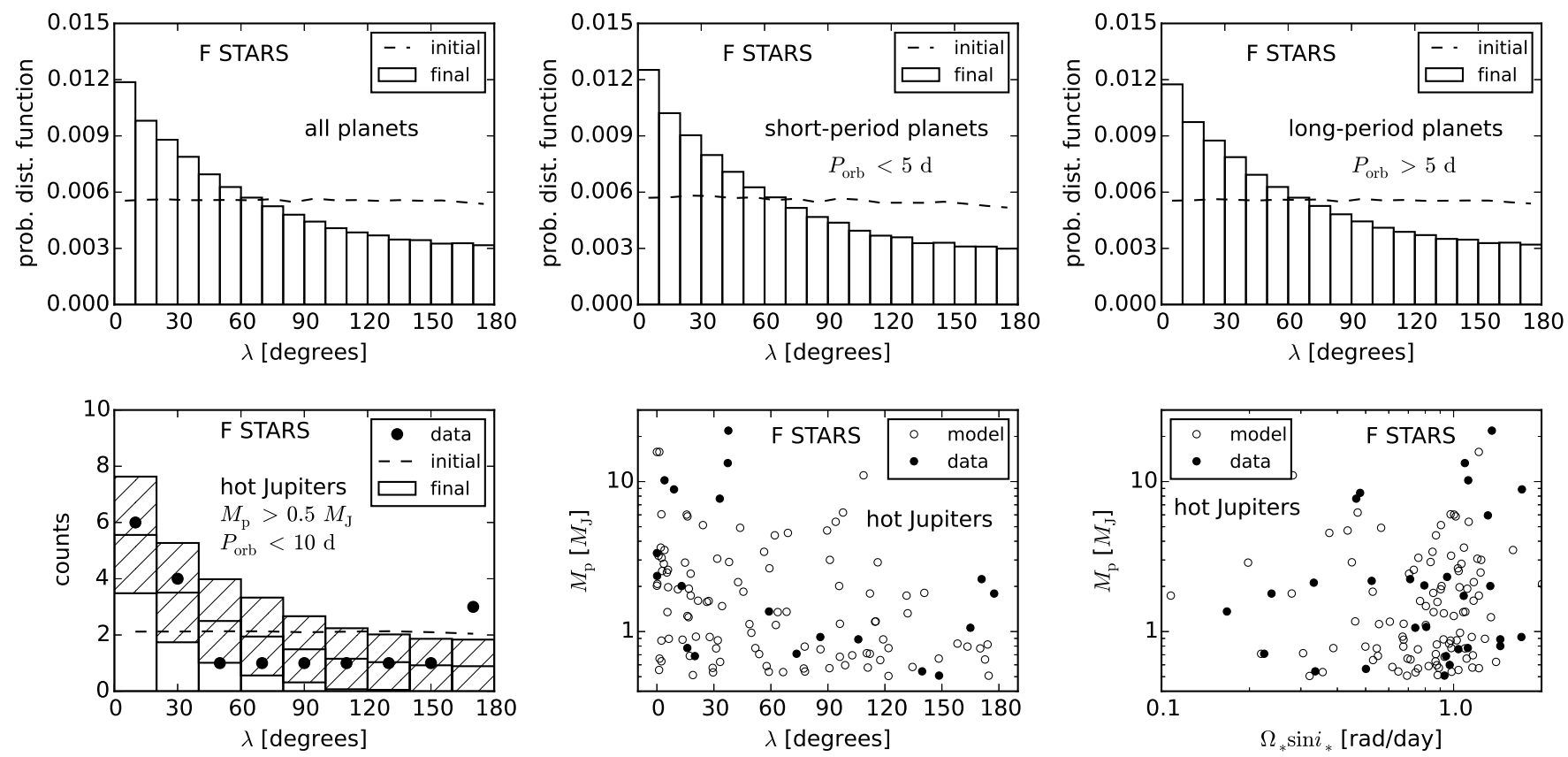

FIG. 2.- Same as Fig. 1 but for the hot-star model.

Batygin, K., \& Adams, F. C. 2013, ApJ, 778, 169

Beaugé, C., \& Nesvorný, D. 2012, ApJ, 751, 119

Ciardi, D. R., van Eyken, J. C., Barnes, J. W., et al. 2015, ArXiv e-prints, arXiv:1506.08719

Cranmer, S. R., \& Saar, S. H. 2011, ApJ, 741, 54

Crida, A., \& Batygin, K. 2014, A\&A, 567, A42

Crockett, C. J., Mahmud, N. I., Prato, L., et al. 2012, ApJ, 761, 164

Dawson, R. I. 2014, ApJ, 790, L31

Fabrycky, D., \& Tremaine, S. 2007, ApJ, 669, 1298
Fabrycky, D. C., \& Winn, J. N. 2009, ApJ, 696, 1230

Fielding, D. B., McKee, C. F., Socrates, A., Cunningham, A. J., \& Klein, R. I. 2015, MNRAS, 450, 3306

Fogg, M. J., \& Nelson, R. P. 2007, A\&A, 472, 1003

Gallet, F., \& Bouvier, J. 2013, A\&A, 556, A36

Greaves, J. S., Kennedy, G. M., Thureau, N., et al. 2014,

MNRAS, 438, L31

Hébrard, G., Ehrenreich, D., Bouchy, F., et al. 2011, A\&A, 527, L11

Herbst, W., \& Mundt, R. 2005, ApJ, 633, 967 
Ida, S., \& Lin, D. N. C. 2004, ApJ, 616, 567

Jackson, B., Barnes, R., \& Greenberg, R. 2009, ApJ, 698, 1357

Johns-Krull, C. M. 2007, ApJ, 664, 975

Königl, A. 1991, ApJ, 370, L39

Kraft, R. P. 1967, ApJ, 150, 551

Lai, D. 2012, MNRAS, 423, 486

-. 2014, MNRAS, 440, 3532

Lai, D., Foucart, F., \& Lin, D. N. C. 2011, MNRAS, 412, 2790

Lin, D. N. C., Bodenheimer, P., \& Richardson, D. C. 1996, Nature, 380, 606

Long, M., Romanova, M. M., \& Lovelace, R. V. E. 2005, ApJ, 634,1214

Mandell, A. M., Raymond, S. N., \& Sigurdsson, S. 2007, ApJ, 660, 823

Matt, S. P., Brun, A. S., Baraffe, I., Bouvier, J., \& Chabrier, G. 2015, ApJ, 799, L23

Mazeh, T., Perets, H. B., McQuillan, A., \& Goldstein, E. S. 2015, ApJ, 801, 3

Meibom, S., Mathieu, R. D., Stassun, K. G., Liebesny, P., \& Saar, S. H. 2011, ApJ, 733, 115

Miller, A. A., Irwin, J., Aigrain, S., Hodgkin, S., \& Hebb, L. 2008, MNRAS, 387, 349

Ogilvie, G. I. 2014, ARA\&A, 52, 171
Rice, W. K. M., Armitage, P. J., \& Hogg, D. F. 2008, MNRAS, 384, 1242

Rogers, T. M., \& Lin, D. N. C. 2013, ApJ, 769, L10

Rogers, T. M., Lin, D. N. C., \& Lau, H. H. B. 2012, ApJ, 758, L6

Spalding, C., \& Batygin, K. 2014, ApJ, 790, 42

Teitler, S., \& Königl, A. 2014, ApJ, 786, 139

Thommes, E. W., Matsumura, S., \& Rasio, F. A. 2008, Science, 321,814

Trilling, D. E., Lunine, J. I., \& Benz, W. 2002, A\&A, 394, 241

Valsecchi, F., Rappaport, S., Rasio, F. A., Marchant, P., \& Rogers, L. A. 2015, ArXiv e-prints, arXiv:1506.05175

Valsecchi, F., \& Rasio, F. A. 2014, ApJ, 786, 102

van Eyken, J. C., Ciardi, D. R., von Braun, K., et al. 2012, ApJ, 755,42

Walkowicz, L. M., \& Basri, G. S. 2013, MNRAS, 436, 1883

Watson, C. A., Littlefair, S. P., Diamond, C., et al. 2011, MNRAS, 413, L71

Weiss, L. M., Marcy, G. W., Rowe, J. F., et al. 2013, ApJ, 768, 14

Winn, J. N., Fabrycky, D., Albrecht, S., \& Johnson, J. A. 2010, ApJ, 718, L145

Xue, Y., Suto, Y., Taruya, A., et al. 2014, ApJ, 784, 66

Youdin, A. N. 2011, ApJ, 742, 38 